\title{
Percepción del Peso de una Carga Según Composición Corporal en Asistentes de Buses Interurbanos
}

\section{PERCEPTION OF THE WEIGHT OF A CARGO ACCORDING TO BODY COMPOSITION IN INTERURBAN BUS ASSISTANTS}

\author{
Eduardo Navarrete Espinoza', Estefanía Saldias Lizama² \\ 1.Académico e Investigador, Escuela de Ciencias y Tecnologías, Campus Los Ángeles, Universidad de Concepción, Los Ángeles, Chile. \\ 2.Ingeniera en Prevención de Riesgos, Licenciada en Ciencias de la Prevención de Riesgos. Los Ángeles, Chile.
}

\section{RESUMEN}

Con el objetivo de determinar la cantidad de peso correspondiente a distintas categorías lingüísticas según la percepción de la carga manipulada por trabajadores y en función de su composición corporal, se realizó un estudio en asistentes de buses interurbanos. Las variables estudiadas fueron: composición corporal expresada en términos de complexión física, esfuerzo percibido a través de la escala de Borg y prueba de confort mediante el diagrama de Corlett y Bishop. Los resultados indicaron que para la complexión física clasificada como estándar, los trabajadores consideraron como peso moderado 19,6 kg; para falta de ejercicios, un peso de 14,3 kg y para obesa, una cantidad de 10,5 kg; lo cual muestra una relación directa entre la composición corporal y la percepción del peso de la carga manipulada, ya que a medida que la complexión física es menos saludable la capacidad de levantar una carga disminuye significativamente. En esta investigación se comparan los resultados obtenidos con los límites de peso establecidos por la Ley № 20.949 que regula en Chile el peso máximo de carga humana, con lo cual se demuestra que el peso percibido, incluso en la categoría extremadamente pesado, se enmarca dentro de la ley actual.

(Navarrete E, Saldías E, 2018. Percepción del Peso de una Carga Según Composición Corporal en Asistentes de Buses Interurbanos. Cienc Trab. Ene-Abr; 20 [61]: 7-13).

Palabras clave: MANEJO MANUAL DE CARGA, PSICOFÍSICA, COMPOSICIÓN CORPORAL, LEY 20.949.

\section{ABSTRACT}

In order to determine the amount of weight corresponding to different linguistic categories according to the perception of the burden handled by workers and based on their body composition, a study was carried out on interurban bus attendants. The variables studied were: body composition expressed in terms of physical constitution, perceived effort through the Borg scale and comfort test using the Corlett and Bishop diagram. The results indicated that for the physical constitution classified as standard, the workers considered as moderate weight $19.6 \mathrm{~kg}$; for the lack of exercises constitution, a weight of $14.3 \mathrm{~kg}$ and for obese constitution, an amount of $10.5 \mathrm{~kg}$; which shows a direct relationship between the body composition and the perception of the weight of the manipulated load, since as the physical complexion is less healthy the ability to lift a load decreases significantly. This research compares the results obtained with the weight limits established by Law No. 20,949, which regulates the maximum weight of burden for humans to carry in Chile, which shows that the perceived weight, even in the extremely heavy category, is framed within of the current law.

Key words: BURDEN HANDLING, PSYCHOPHYSICS, BODY COMPOSITION, LAW 20,949.

\section{INTRODUCCIÓN}

En los países industrializados, alrededor de un tercio de los días laborales perdidos relacionados con problemas de salud se deben a trastornos musculoesqueléticos, y de los cuales, un 60\% se relaciona con problemas localizados en la espalda. ${ }^{1}$ Un trastorno musculoesquelético relacionado con el trabajo es una lesión de los

\section{Correspondencia / Correspondence:}

Eduardo Navarrete Espinoza

Universidad de Concepción

Escuela de Ciencias y Tecnologias, Campus Los Ángeles

Juan Antonio Coloma 0201, Los Ángeles, Chile

Tel. (56-43) 2405217

e-mail: ednavarr@udec.cl

Recibido??? músculos, tendones, ligamentos, nervios, articulaciones, cartílagos, huesos o vasos sanguíneos de los brazos, piernas, cabeza, cuello o espalda que se produce o se agrava por tareas laborales como levantar, empujar o jalar objetos. ${ }^{2}$ A nivel mundial, 37\% del dolor lumbar se atribuyó a la ocupación, la proporción atribuible fue mayor en hombres que en mujeres, debido a la mayor participación en la fuerza laboral y en ocupaciones relacionadas con levantar objetos pesados o vibración de todo el cuerpo. ${ }^{3}$

De acuerdo a datos estadísticos de la Organización Internacional del Trabajo (OIT), se señala que cerca del 25\% del total de accidentes laborales son originados por el manejo manual de carga $(\mathrm{MMC})^{4}$, lo cual se define como cualquier labor que requiera principalmente el uso de fuerza humana para levantar, sostener, colocar, empujar, portar, desplazar, descender, transportar o ejecutar cualquier otra acción que permita poner en movimiento o detener un objeto. A su vez, se entiende como carga a cualquier objeto, animado o inanimado, que se requiera mover utilizando fuerza humana, y cuyo peso supere los 3 kilógramos. $^{5}$ 
En Chile, los organismos administradores de la Ley $\mathrm{N}^{\circ} 16.744$ del Seguro contra Accidentes del Trabajo y Enfermedades Profesionales revelan en sus estadísticas que el dolor lumbar o lumbago, producto de sobre-esfuerzos físicos, representa el tercer lugar en términos de días totales de tratamiento o número de casos. Además, menciona que si se consideran todos los diagnósticos de trastornos musculoesqueléticos dorso-lumbares, donde el MMC podría ser uno de los factores causales, los diagnósticos de lumbago, desgarro paravertebral y dorsalgia constituyen el 97,3\% de las lesiones. Por otra parte, el $80 \%$ de esos casos afecta a hombres y el 63,5\% de las lesiones reportadas ocurren en trabajadores entre 25 y 44 años de edad. ${ }^{5}$

Si bien en Chile fue promulgada en el año 2005 la Ley 20.001, que regula el peso máximo de carga humana, en esta solo se mencionaba que si la manipulación era manual, no se permitiría que se operara con cargas superiores a 50 kilógramos en el caso de los hombres. Dicha ley fue modificada el año 2016, a través de la Ley 20.949, disminuyendo a 25 kilógramos el límite máximo de carga permisible. ${ }^{6}$ Sin embargo, existe un vacío en lo que respecta a cómo perciben los trabajadores el peso de la carga que manipulan manualmente. La percepción es la imagen mental que se forma con ayuda de la experiencia y necesidades, es resultado de un proceso de selección, interpretación y corrección de sensaciones. Debido a lo anterior, es importante tener en cuenta factores que influyen en la percepción; algunos de ellos son, por ejemplo, el porcentaje de masa grasa (PMG), como lo ratifican Waddell y Burton ${ }^{7}$, quienes mencionan que existen factores personales y no-laborales que podrian propiciar la generación de lesiones lumbares en los ambientes de trabajo. Algunos de esos factores son el índice de masa corporal (IMC), porcentaje de masa grasa (PMG), capacidad aeróbica, hábito de consumo de tabaco, edad, género, experiencia y habilidades, umbral personal, entre otros; existiendo, además, una relación dosis-respuesta que puede variar ampliamente entre distintas personas. ${ }^{4}$

Teniendo en cuenta lo anterior, es importante estudiar la composición corporal como uno de los factores influyentes en la percepción, debido a que es el único factor sobre el cual no se ha realizado estudios. Algunos de los mecanismos utilizados para conocer la composición corporal son la antropometría (menos invasiva y de fácil obtención de datos); sin embargo, esta técnica no es muy exacta; otro mecanismo que se ha desarrollado durante las últimas décadas es la bioimpedancia eléctrica (BIA), siendo una forma rápida, simple, segura y relativamente económica de cuantificar los componentes de la composición corporal. La bioimpedancia eléctrica se basa sobre el principio de que los tejidos ricos en agua y electrolitos oponen una menor resistencia al paso de una corriente eléctrica, a diferencia del tejido adiposo, rico en lípidos, que opone una mayor resistencia. Por lo tanto, su utilización permite el cálculo del volumen del agua corporal y, a partir de ésta, puede determinarse la masa magra (masa libre de grasa), como la diferencia entre el peso corporal y la masa grasa. ${ }^{8}$

La norma ISO 11.228 referente al MMC menciona que la estimación del riesgo al manipular cargas considera capacidades de tipo Biomecánica, Fisiológica y Psicofísica, siendo esta última la que considera la percepción del trabajador respecto del: esfuerzo, fuerza e incomodidad aceptables. Por lo tanto, el enfoque psicofísico se relaciona directamente con la percepción de la carga de trabajo físico. ${ }^{9}$ En el ámbito de la psicofísica aplicada al MMC, se utilizan categorías lingüísticas tales como muy liviana, liviana, pesada o muy pesada para calificar la carga de trabajo. En este mismo contexto, normalmente se utilizan expresiones como aceptable o moderado para calificar el peso de los objetos que estaría dentro de rangos seguros o tolerables para los trabajadores. $1^{0} \mathrm{~A}$ pesar de lo anterior, en Chile aún no se han realizado estudios desde el ámbito psicofísico para determinar los límites de pesos aceptables, por lo que sólo se han obtenido datos de poblaciones extranjeras, tanto de Europa como de Norteamérica, lo cual es cuestionable. ${ }^{11}$

Tradicionalmente, el nivel de exigencia física de las actividades de MMC es evaluado comparando las demandas de la labor con las capacidades humanas descritas en la literatura científica. Si esas demandas exceden las capacidades humanas, la tarea es considerada riesgosa. El riesgo de lesionarse aumenta mientras más grande es esta diferencia. ${ }^{12}$ Por tal motivo, es importante conocer la cantidad de peso en kilógramos que es considerado seguro para los trabajadores, de tal manera que ejecuten su trabajo de manera segura y tolerable. Una tarea se considera aceptable, cuando es capaz de realizarla al menos el 90\% de la población trabajadora; si solo la puede realizar entre el 90\% y el 75\%, la tarea debe ser mejorada; y si puede ser realizada por menos del 75\% de los trabajadores, se considera de riesgo y debe ser rediseñada. ${ }^{13}$

Según la Guía Técnica para la Evaluación del Trabajo Pesado ${ }^{14}$, el riesgo dorso-lumbar presenta las tasas más altas en los rubros de transporte-almacenaje-comunicaciones (12,2\%), construcción $(10,9 \%)$ y manufactura $(10,6 \%)$. El transporte comercial se refiere a todos los medios e infraestructuras implicadas en el movimiento de las personas o bienes, así como los servicios de recepción, entrega y manipulación de tales bienes. El transporte comercial de personas se clasifica como servicio de pasajeros, y el de bienes, como servicio de mercancías. Precisamente es en el transporte de pasajeros donde el personal a cargo de subir y bajar el equipaje de los pasajeros del bus, comúnmente llamado auxiliar de buses, es el que se encuentra directamente expuesto a riesgos de dolor lumbar, al manipular cargas que exceden sus capacidades físicas. ${ }^{15}$

El objetivo general de este estudio fue determinar la cantidad de peso en kilógramos que auxiliares de buses de un terminal de la comuna de Los Ángeles, Chile, perciben para cada categoría lingüística de la Escala de Borg, de acuerdo a su composición corporal. Los objetivos específicos propuestos fueron determinar la composición corporal de la muestra de trabajadores del transporte de pasajeros; determinar la cantidad de peso según su percepción, correspondiente a cada categoría lingüística (Escala de Borg); relacionar la percepción del peso de una carga con las variables de composición corporal analizadas; comparar la percepción de los trabajadores respecto al manejo manual de carga (kg) con la legislación chilena vigente (Ley 20.949); establecer los límites de peso recomendables en manejo manual de carga para los auxiliares de buses; y finalmente, evaluar las dolencias musculoesqueléticas presentadas por los trabajadores.

\section{MATERIALES Y MÉTODOS}

La presente investigación se basó en un diseño no experimental, de tipo transversal, descriptivo y correlacional. La población bajo estudio correspondió a trabajadores del transporte público de pasajeros, específicamente asistentes de buses interurbanos de la comuna de Los Ángeles, región del Biobío, Chile.

\section{Muestra}

La muestra estuvo compuesta por 30 asistentes de buses de género masculino de un terminal de la comuna de Los Ángeles, quienes desempeñaban sus funciones desde la ciudad hacia las distintas comunas de la Provincia del Biobío. El rango de edad considerado 
fue de 18 a 60 años, debido a que el 63,5\% de las lesiones reportadas a los organismos administradores de la Ley 16.744 ocurren en trabajadores entre 25 y 44 años de edad. ${ }^{5}$

\section{Criterios de inclusión}

Los trabajadores formaron parte de la investigación voluntariamente, firmando para tales efectos un consentimiento informado, en el cual se detallaba el objetivo del estudio y la confidencialidad de los datos. Se excluyeron aquellos asistentes de buses que llevaban desempeñando sus funciones por un período menor a cuatro meses, que utilizaran marcapasos o cualquier otro tipo de implante mecánico, que presentaran alergias a los metales y que fueran de género femenino.

\section{Descripción del estudio}

La obtención de los datos de composición corporal se realizó en condiciones ambientales homologables, utilizando dependencias correspondientes a la zona de custodia dispuesta en el propio terminal de buses. Se procedió a registrar los datos de los participantes y las variables de interés (antigüedad laboral, edad, peso, talla, entre otras); todas las medidas se realizaron según los procedimientos y técnicas descritas por Lohman et $\mathrm{al}^{16}$, por lo que cada trabajador se pesó descalzo, de pie, con el cuerpo erguido en máxima extensión, cabeza recta y con ropa ligera, de modo de no sobreestimar la medición. La obtención de datos se realizó durante la jornada de la mañana, en los horarios de 8:30 horas a 12:00 horas, asegurando, para efectos del estudio, que los trabajadores evaluados no presentaran fatiga. Al momento de realizar las mediciones cada auxiliar fue sometido a levantar manualmente una carga determinada de peso, sosteniendo para ello un contenedor de madera con ambas manos, a una posición de la carga más favorable, es decir, pegada al cuerpo, a una altura comprendida entre los codos y los nudillos ${ }^{17}$, en la cual el trabajador manifestó la percepción sobre su esfuerzo físico y se ajustó el peso, hasta un nivel que consideró aceptable o moderado.

\section{METODOLOGÍA}

\section{Instrumentos}

Para la medición de talla y peso se utilizó una báscula digital con tallímetro marca TANITA WB3000, con una precisión de $1 \mathrm{~mm}$ y 50 gramos, respectivamente. La determinación de la composición corporal se hizo a través de bioimpedancia eléctrica, para lo cual se utilizó el analizador de composición corporal TANITA SC331S.

Para determinar los valores de las categorías lingüísticas según la escala de Borg, de acuerdo a la percepción de cada trabajador sobre el esfuerzo percibido al manipular la carga, se fabricó un contenedor de madera con perforaciones en cada costado, a modo de asas, localizadas en el tercio superior de su dimensión vertical11, al cual se le incorporó peso progresivamente, correspondiente a bloques de concreto de peso específico (1 kilógramo y 2 kilógramos).

\section{Procedimiento}

\section{Composición corporal}

La composición corporal se determinó a través de bioimpedancia, realizándose las mediciones de acuerdo al procedimiento del manual de instrucciones del analizador de composición corporal SC-331S. La clasificación de la composición corporal se expresó en términos de complexión física (Tabla 1).
Tabla 1.

Clasificación composición corporal.

\begin{tabular}{|c|c|c|}
\hline Resultado & $\begin{array}{l}\text { Complexión } \\
\text { física }\end{array}$ & Explicación \\
\hline 1 & Preobesa & Obeso con contextura pequeña \\
\hline 2 & Obesa & Obeso con contextura mediana \\
\hline 3 & Complexión robusta & Obeso con contextura grande \\
\hline 4 & Falta de ejercicios & $\begin{array}{l}\text { Masa muscular baja y porcentaje de grasa } \\
\text { corporal promedio }\end{array}$ \\
\hline 5 & Estándar & $\begin{array}{l}\text { Masa muscular alta y porcentaje de grasa } \\
\text { corporal promedio }\end{array}$ \\
\hline 6 & Musculosa estándar & $\begin{array}{l}\text { Masa muscular promedio y porcentaje de } \\
\text { grasa corporal promedio (atleta) }\end{array}$ \\
\hline 7 & Delgada & Masa muscular baja y grasa corporal baja \\
\hline 8 & Delgada y musculosa & Delgado y musculoso (atleta) \\
\hline 9 & Muy musculosa & Muy musculoso (atleta) \\
\hline
\end{tabular}

\section{Metodología Snook y Ciriello}

Para obtener datos sobre la carga manipulada, y de acuerdo al enfoque psicofísico $^{11}$, se utilizó la metodología Snook y Ciriello ${ }^{18}$, la cual permite determinar los pesos máximos aceptables para diferentes acciones, como el levantamiento, el descenso, el empuje, el arrastre y el trasporte de cargas. Según Ayoub y Dempsey ${ }^{19}$, el enfoque psicofisico aplicado al MMC permite una razonable simulación del trabajo industrial, los resultados son reproducibles y se relacionan con el dolor lumbar en tareas de MMC. Los pesos aceptables de levantamiento de carga determinados ocupando este enfoque, integran las demandas biomecánicas y fisiológicas impuestas por estas tareas.

El peso máximo aceptable, de acuerdo a la metodología Snook y Ciriello $^{18}$, corresponde al mayor peso que una persona puede levantar a una frecuencia dada y durante un tiempo determinado, sin llegar a estresarse o a cansarse excesivamente. Los pesos máximos aceptables se determinan para cinco percentiles (10, 25, 50, 75 y 90), que indican los pesos máximos permitidos para que la acción sea segura, para el 10\%, 25\%, 50\%, 75\% y 90\% de la población. Para los valores introducidos no tabulados en las tablas se consultan los valores tabulados más próximos. En el caso de la frecuencia, si el valor introducido no se encuentra tabulado, el peso máximo aceptable se obtiene por interpolación lineal de las frecuencias tabuladas entre las que se encuentra la introducida.

\section{Diagrama de Corlett y Bishop}

El Diagrama de Corlett y Bishop es una prueba de confort basada en la inspección de las partes del cuerpo, donde el trabajador localiza el lugar en que se manifiestan las molestias. ${ }^{20}$ Para la inspección de dolor de las partes del cuerpo se mostró a los trabajadores un mapa corporal, en el cual señalaron las zonas exactas de sus molestias.

\section{Escala de Borg del esfuerzo percibido}

El esfuerzo percibido es una valoración subjetiva que indica la opinión del sujeto respecto a la intensidad del trabajo realizado21. Esta escala cuantifica la percepción del esfuerzo en 20 niveles, utilizándose para efectos de este estudio, solo seis (Tabla 2), siendo las categorias Moderada y Pesada las de mayor interés.

Tabla 2.

Escala del esfuerzo percibido (Borg CR-10).

$\begin{array}{lc}\text { Categoría Lingüistica } & \text { Código numérico } \\ \text { Muy Liviano (ML) } & 1 \\ \text { Liviano (L) } & 2 \\ \text { Moderado (M) } & 3 \\ \text { Pesado (P) } & 5 \\ \text { Muy Pesado (MP) } & 7 \\ \text { Extremadamente pesado (EP) } & 10\end{array}$


Antecedentes legales

Para el presente estudio se analizó la Ley 20.949 del Ministerio del Trabajo y Previsión Social, la cual fue publicada en el Diario Oficial con fecha 16 de septiembre de 2016, con la finalidad de contrastar la cantidad de peso según la percepción que se obtuvo de la investigación. Según la modificación del Artículo 211-H de la Ley 20.001, si la manipulación manual es inevitable y las ayudas mecánicas no pueden usarse, no se permitirá que se opere con cargas superiores a 25 kilógramos. $^{6}$

\section{Análisis estadístico}

Se realizó un análisis descriptivo de las variables en estudio, obteniendo medidas de tendencia central y dispersión, además de las distribuciones de probabilidad de los datos. La evaluación del efecto de la complexión física sobre la percepción de peso se realizó a través de un análisis de varianza. En el caso de encontrarse diferencias entre las distintas categorias de complexión, estas fueron identificadas a través del test de Tukey $(p=0,05)$. Las variables de composición corporal y percepción de peso aceptable de levantamiento en manipulación manual de carga se relacionaron a través de un análisis de correlación. Dado que la edad no presentó normalidad, se realizó una matriz de correlación no paramétrica (Spearman). Los análisis se realizaron con el software Statistica 10.0.

\section{RESULTADOS}

\section{Caracterización sociodemográfica}

La muestra estuvo compuesta por 30 trabajadores del género masculino, quienes presentaron un promedio en edad de 30,5 \pm 10,81 años y una antigüedad en el puesto de trabajo de 4,8 \pm 5,7 años.

\section{Composición corporal}

Los trabajadores presentaron en promedio un peso de 80,8 \pm 14,29 $\mathrm{kg}$ y talla de $169,0 \pm 7,65 \mathrm{~cm}$. El porcentaje de masa grasa (MG) determinado a través de bioimpedancia eléctrica fue de 25,3 \pm $7,05 \%$ y un porcentaje de masa muscular (MM) de 70,9 $\pm 6,61 \%$. Se presentó una distribución por rangos clasificatorios, de un 73\% de personas en categoría obesa, 10\% en falta de ejercicios y 17\% estándar (Figura 1).

\section{Percepción de la carga manipulada}

De acuerdo a la percepción del peso de la carga manipulada, en la Tabla 3 se observa que el promedio para la categoría moderada (M) fue de $12,4 \pm 4,04 \mathrm{~kg}$, pesado $(\mathrm{P})$ de $16,9 \pm 4,99 \mathrm{~kg}$, muy pesado (MP) de $21,1 \pm 5,05 \mathrm{~kg}$ y para extremadamente pesado (EP) de 24,0

\section{Figura 1.}

Clasificación nutricional según bioimpedancia eléctrica.

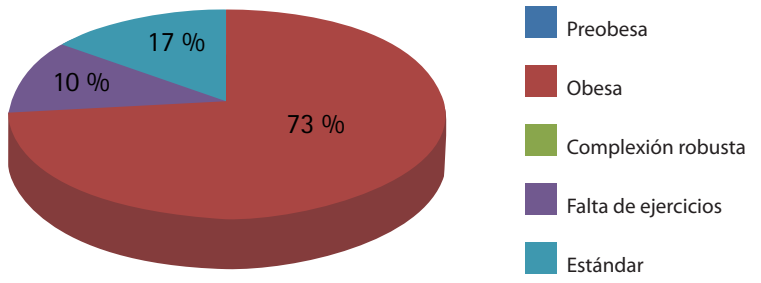

Tabla 3.

Valores promedios de la percepción de la carga manipulada con sus respectivas desviaciones estándar $(n=30)$.

$\begin{array}{ccc}\text { Percepción de la carga } & \text { Media }(\mathrm{kg}) & \text { D.E. } \\ \text { ML } & 6,5 & 2,64 \\ \text { L } & 8,8 & 3,29 \\ \text { M } & 12,4 & 4,04 \\ \text { P } & 16,9 & 4,99 \\ \text { MP } & 21,1 & 5,05 \\ \text { EP } & 24,0 & 4,21\end{array}$

Con D.E. = desviación estándar; $\mathrm{ML}=$ muy liviano; $\mathrm{L}=$ liviano; $\mathrm{M}=$ moderado;

$\mathrm{P}=$ pesado; $\mathrm{MP}=$ muy pesado; $\mathrm{EP}=$ extremadamente pesado.

$\pm 4,21 \mathrm{~kg}$, lo cual indica que el peso de la carga que los trabajadores perciben en categorías pesadas, e incluso extremadamente pesada, está acorde con lo establecido por la legislación chilena vigente sobre el tema, la cual menciona que si la manipulación es manual, no se permitirá la operación con cargas superiores a 25 kilógramos en el caso de los hombres.

Percepción del peso de la carga según composición corporal Para la complexión física clasificada como estándar, los trabajadores consideraron como peso moderado (M) 19,6 kg; para falta de ejercicios, un peso de $14,3 \mathrm{~kg}$ y para obesa, una cantidad de 10,5 kg; lo cual muestra una relación entre la complexión física y la percepción del peso de la carga manipulada, ya que a medida que la complexión física es menos saludable, la capacidad de levantar una carga disminuye significativamente; manifestándose del mismo modo, esta tendencia para las demás categorias lingüísticas. En todas las categorías lingüísticas se encontraron diferencias significativas en la percepción de peso, entre las complexiones estándar y obesa (Tabla 4).

Tabla 4.

Valores promedios de percepción de peso $(\mathrm{kg})$ para cada categoria lingüística según complexión física.

\begin{tabular}{|c|c|c|c|c|c|c|}
\hline \multicolumn{7}{|c|}{ Complexión física } \\
\hline $\begin{array}{l}\text { Categoria } \\
\text { lingüistica }\end{array}$ & Estándar & D.E & $\begin{array}{l}\text { Falta de } \\
\text { ejercicios }\end{array}$ & D.E & Obesa & D.E \\
\hline$\widehat{M L}$ & 10,2 a & 3,35 & $6,0 \mathrm{~b}$ & 2,00 & $5,7 \mathrm{~b}$ & 1,78 \\
\hline L & 13,6 a & 3,65 & $9,7 a b$ & 2,89 & 7,6 b & 2,15 \\
\hline $\mathrm{M}$ & 19,6 a & 3,65 & $14,3 \mathrm{~b}$ & 1,15 & $10,5 \mathrm{c}$ & 1,79 \\
\hline$P$ & 24,6 a & 4,16 & $19,0 a b$ & 3,00 & $14,9 \mathrm{~b}$ & 3,33 \\
\hline MP & 27,4 a & 3,58 & $23,0 a b$ & 5,20 & $19,5 b$ & 4,17 \\
\hline EP & 28,8 a & 2,17 & $25,0 a b$ & 5,20 & $22,8 \mathrm{~b}$ & 3,74 \\
\hline
\end{tabular}

En filas, valores con letras iguales no difieren estadisticamente, con $p>0,05$ (Test de Tukey). Con $\mathrm{D} . \mathrm{E}$ = desviación estándar; $\mathrm{ML}=$ muy liviano; $\mathrm{L}=$ liviano; $\mathrm{M}=$ moderado; $\mathrm{P}=$ pesado; $\mathrm{MP}=$ muy pesado; $\mathrm{EP}=$ extremadamente pesado.

Relación entre categorias lingüisticas de percepción de peso y variables de composición corporal

Las categorías de percepción de peso muy liviano (ML), liviano (L), moderado $(\mathrm{M})$, pesado $(\mathrm{P})$ y muy pesado $(\mathrm{MP})$ se relacionaron significativamente con las variables de composición corporal porcentaje de masa grasa (MG) y porcentaje de masa muscular (MM), evidenciándose que a mayor porcentaje de masa grasa, menor es la cantidad de peso que puede manipular un trabajador (Tabla 5). Por su parte, trabajadores con un porcentaje de masa muscular (MM) mayor, el peso de carga capaz de manipular es significativamente mayor (Figura 3).

A medida que aumenta el porcentaje de masa grasa en los trabajadores, el peso de la carga percibida como moderada, disminuyó significativamente $(\mathrm{R} 2=0,519$; valor $\mathrm{p}=0,005)$ (Figura 2); en cambio, al aumentar el porcentaje de masa muscular, aumenta también el peso de la carga percibida como moderada $(\mathrm{R} 2=0,523$; valor $\mathrm{p}=0,005$ ) (Figura 3). 
Figura 2.

Relación porcentaje masa grasa (MG) y percepción del peso moderado.

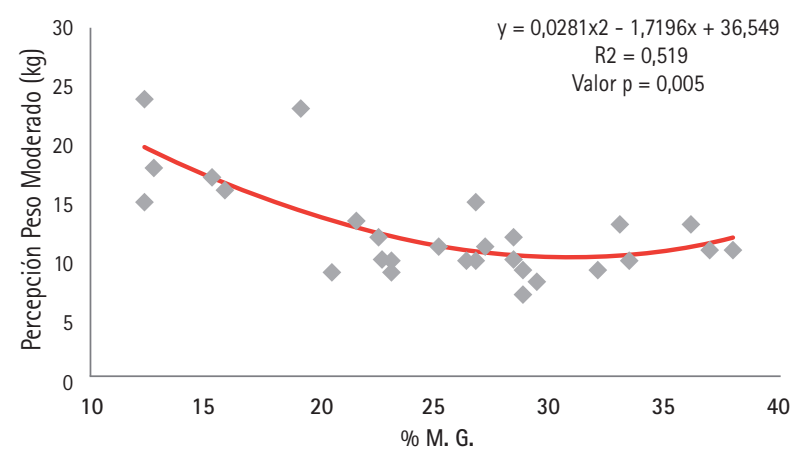

Figura 3.

Relación porcentaje masa muscular (MM) y percepción del peso moderado.

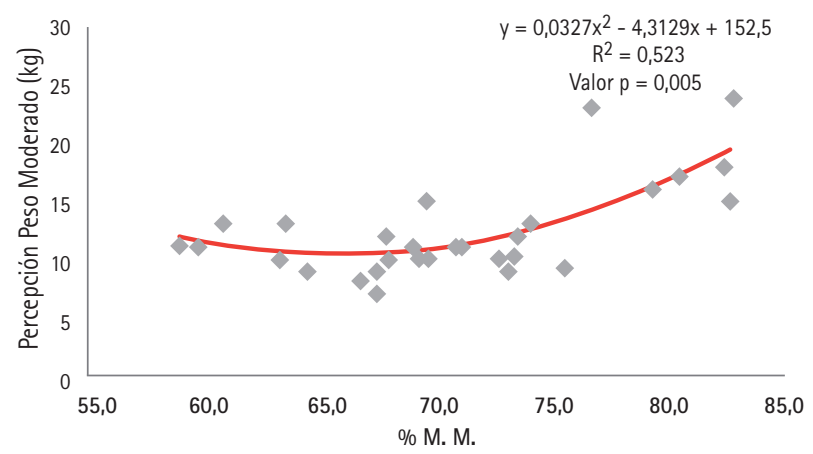

Las categorias de percepción de peso muy liviano (ML), liviano (L) y extremadamente pesado (EP), se relacionaron estadísticamente, además, con la edad, determinándose que la cantidad de peso que pueden manipular los trabajadores disminuye significativamente $(\mathrm{p}<$ $0,05)$ a medida que aumenta la edad (Tabla 5).

\section{Determinación del peso recomendable en manejo manual de carga para los auxiliares de buses}

Debido a las características del puesto de trabajo bajo estudio, el peso máximo aceptable para realizar las tareas de levantamiento de carga corresponde a 18,33 $\mathrm{kg}$ (considerando un porcentaje de protección del 90\% de los trabajadores evaluados). Dado que el promedio de carga manipulada es de $15 \mathrm{~kg}$ y el máximo aceptable para levantamiento es de $18,33 \mathrm{~kg}$, y que al configurar la forma de la carga esta no cuenta con asas, este valor se modifica, estableciéndose que el peso aceptable se reduce de 18,33 kg a 15,58 kg;

\section{Tabla 5.}

Correlaciones entre la edad, variables de composición corporal y categorias lingüisticas de percepción de peso según la escala de Borg.

$\begin{array}{lcccccc}\text { Variables } & \text { ML } & \text { L } & \text { M } & \text { P } & \text { MP } & \text { EP } \\ \text { Edad (años) } & -0,45^{*} & -0,43^{*} & -0,32 n . s & -0,34 n . s & -0,31 n . s & -0,38^{*} \\ \text { Peso (kg) } & -0,32 n . s & -0,18 n . s & -0,27 n . s & -0,28 n . s & -0,24 n . s & -0,24 n . s \\ \text { Talla (cm) } & -0,01 \text { n.s } & 0,12 n . s & 0,08 n . s & 0,07 n . s & 0,02 n . s & 0,01 n . s \\ \text { MG (\%) } & -0,53^{* *} & -0,47^{* *} & -0,48^{* *} & -0,48^{* *} & -0,39^{*} & -0,35 n . s \\ \text { MM (\%) } & 0,52^{* *} & 0,47^{* *} & 0,49^{* *} & 0,48^{* *} & 0,39^{*} & 0,36 n . s\end{array}$

Con $^{* *}=$ Altamente significativo $(p<0,01){ }^{*}=$ Significativo $(p<0,05)$ y $n . s=$ No significativo ( $p>0,05)$ (Test de Spearman).

Con $M G=$ Masa Grasa; $M M=$ Masa Muscular; $M L=$ muy liviano; $\mathrm{L}=$ liviano; $\mathrm{M}=$ moderado; $\mathrm{P}=$ pesado; $\mathrm{MP}=$ muy pesado; $\mathrm{EP}=$ extremadamente pesado.
Tabla 6.

Determinación del peso recomendable en MMC según la metodología Snook y Ciriello.

\begin{tabular}{ll}
\hline Análisis & Resultado \\
\hline Tipo de acción de manipulación manual de carga evaluada & Levantamiento \\
Peso medio de la carga manipulada por el trabajador & $15 \mathrm{~kg}$ \\
\hline Peso máximo aceptable para la tarea de levantamiento de la carga & $18,33 \mathrm{~kg}$ \\
\hline Peso máximo aceptable para cargas sin asas & $\begin{array}{l}15,58 \mathrm{~kg} \\
\text { (reducción 15\%) }\end{array}$ \\
\hline $\begin{array}{l}\text { Peso máximo aceptable para cargas alejadas del cuerpo } \\
\text { Relación entre el peso medio de la carga y el peso máximo }\end{array}$ & $9,16 \mathrm{~kg}$ \\
aceptable para el levantamiento & (reducción 50\%) \\
\hline
\end{tabular}

no superando el peso máximo aceptable según la metodología de Snook y Ciriello.

\section{Evaluación de dolencias musculoesqueléticas}

Respecto a la presencia de dolencias musculoesqueléticas, se observó una alta frecuencia de malestares a nivel lumbar $(57,7 \%)$ y dorsal $(30,8 \%)$ (Figura 4). Lo anterior es atribuible a posturas de trabajo inadecuadas al realizar MMC durante toda la jornada de trabajo; sumado a esto, se logra observar que existe un porcentaje importante relacionado a dolores en la rodilla izquierda (26,9\%), lo cual se pudo constatar mediante observaciones in situ, que se produce debido a las maniobras que deben realizar los trabajadores al subir a los buses en movimiento, apoyando para ello toda su pierna izquierda.

\section{DISCUSIÓN}

Los resultados en cuanto a la composición corporal de los trabajadores evaluados se explica debido al régimen irregular de alimentación con la que cuentan los auxiliares de buses, basándose principalmente en comida rápida, como causa del escaso tiempo que disponen para alimentarse. Es importante considerar que una alimentación balanceada, con el suministro de nutrientes y calorías acorde con el tipo de trabajo que se realiza, permite una adecuada relación talla/ peso y el aporte energético necesario para la labor. Según Rueda y Zambrano ${ }^{23}$, el control de sobrepeso y obesidad previene molestias en la espalda y el aumento de la presión sobre los discos lumbares. Un trabajador obeso o con sobrepeso, tiene entre tres y cinco veces más riesgo de sufrir lesiones en la espalda. En el MMC, las partes del cuerpo involucradas son las piernas, como propulsor y estabilizador del levantamiento de la carga; la columna vertebral o tronco, como estructura de soporte; y los brazos, como segmentos de sujeción y transmisión de fuerza. La obesidad y la postura encorvada son potenciales condiciones de riesgo para la columna.

Respecto al MMC que realizan los auxiliares de buses se encontraría, de acuerdo a la legislación, en un rango seguro para la realización de la labor. Aunque la puesta en vigencia de esta ley constituye un avance en materia de protección de la salud de los trabajadores, es importante destacar que la carga señalada en ella constituye un límite máximo legal, que no debe ser confundido ni interpretado como un límite seguro para la salud de los trabajadores. ${ }^{11}$ El Instituto Nacional de Seguridad e Higiene en el Trabajo (INSHT) de España establece que cargas que pesen más de $25 \mathrm{~kg}$ muy probablemente constituyan un riesgo en sí mismas, aunque no existan otras condiciones ergonómicas desfavorables; además, menciona que el peso 
Figura 4.

Frecuencias porcentuales de dolencias musculoesqueléticas según diagrama de Corlett y Bishop ${ }^{22}$.

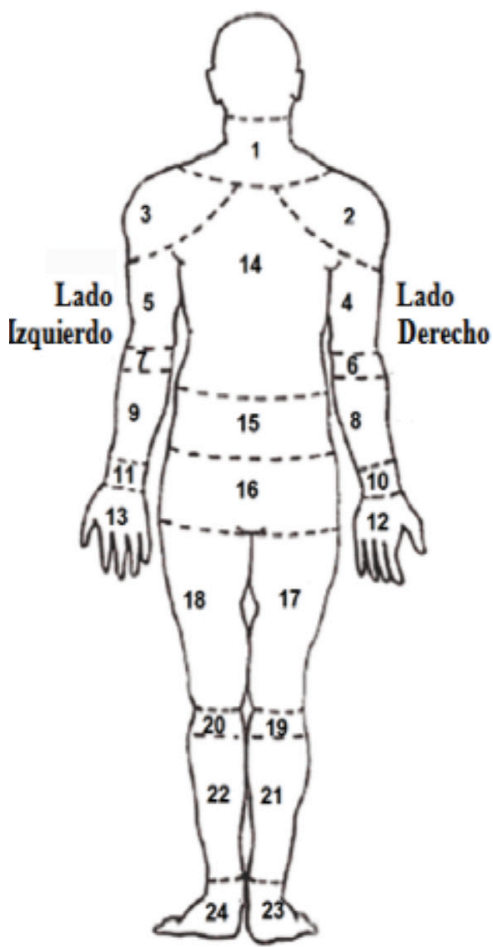

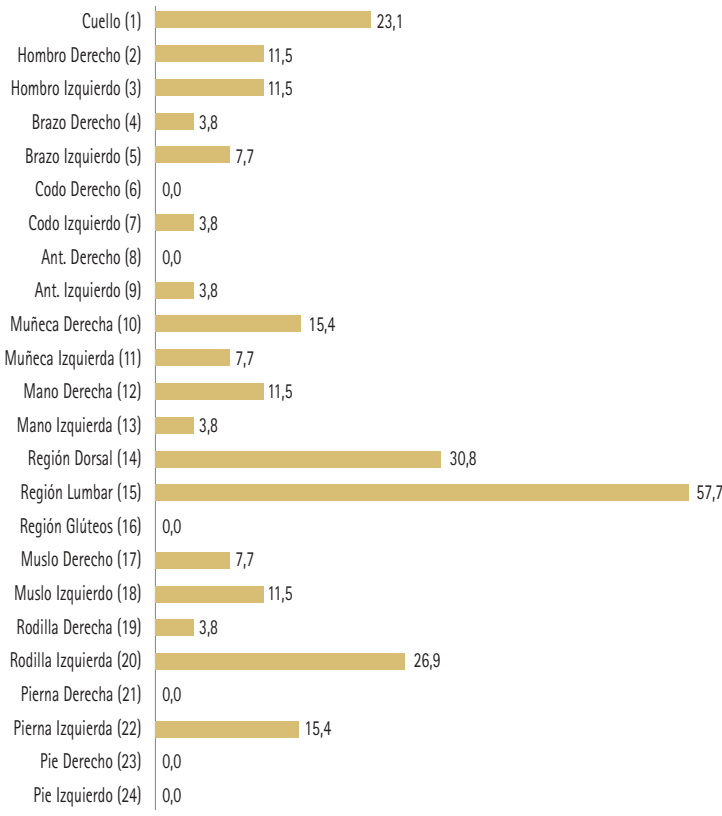

menciona que existe evidencia de que las exigencias laborales que sobrepasan ciertos límites fisiológicos y biomecánicos de las personas están relacionadas con los trastornos musculoesqueléticos, y que para poder identificar y controlar esta relación causaefecto es imprescindible realizar un adecuado estudio de las exigencias y factores de riesgo de las tareas laborales.

En conclusión, el mayor porcentaje de la muestra evaluada se encontró en estado nutricional obeso, de acuerdo al método de bioimpedancia eléctrica. El peso de la carga que los trabajadores percibieron en categorías pesadas, e incluso extremadamente pesada, se enmarca dentro del límite estipulado en la Ley 20.949. La ley 20.001, por su parte, es insuficiente en su planteamiento, ya que estipula la disminución de la manipulación de carga al incorporar elementos mecánicos, el peso máximo y la implementación de capacitación, dejando de lado las condiciones que se deben

máximo que se recomienda no sobrepasar, en condiciones ideales de manipulación, es de $25 \mathrm{~kg}$, protegiendo así al 85\% de la población trabajadora sana. ${ }^{24}$

Según la VI Encuesta Nacional de Condiciones de Trabajo realizada por el INSHT en el año 2007, el 55\% de los trabajadores que declaran manipular cargas pesadas siempre, casi siempre o a menudo, durante su jornada laboral, manifiestan sufrir molestias musculoesqueléticas en la zona lumbar. Estas lesiones, aunque no son mortales, pueden tener larga y difícil curación, y en muchos casos requieren un largo periodo de rehabilitación, originando grandes costes económicos y humanos, ya que el trabajador queda muchas veces incapacitado para realizar su trabajo habitual, y su calidad de vida puede quedar deteriorada. Por lo tanto, se debe analizar las condiciones de trabajo en las que ejecutan sus labores los trabajadores, para lograr establecer las medidas necesarias y evitar que las molestias en la zona lumbar sigan aumentando. De acuerdo a la Organización Mundial de la Salud, las dolencias musculoesqueléticas constituyen una de las principales causas de ausentismo laboral en el mundo. Actualmente, existe evidencia científica que respalda la tesis de que el manejo adecuado de los factores de riesgo relativos a trastornos musculoesqueléticos favorece, no sólo la salud, sino que también la capacidad de trabajo de las personas y, consecuentemente, la productividad, lo que resulta en un mejor funcionamiento del sistema laboral. ${ }^{25}$ Además, tener cuando la manipulación de carga sea inevitable; es decir, no se describen las características de tamaño, agarre, frecuencia y los factores ambientales, que en el caso de este estudio, constituye el factor que se presenta con mayor frecuencia para un manejo manual de carga seguro para la salud del trabajador. Existe una relación estadísticamente significativa entre la complexión física y la percepción del peso de la carga manipulada, determinándose que a medida que la complexión física va en desmedro, la capacidad de levantar una carga disminuye. Las categorias de percepción de peso muy liviano (ML), liviano (L), moderado (M), pesado (P) y muy pesado (MP) se relacionaron significativamente con las variables de composición corporal porcentaje de masa grasa (MG) y porcentaje de masa muscular (MM), evidenciándose que a mayor porcentaje de masa grasa, menor es la cantidad de peso que puede manipular un trabajador. Por su parte, trabajadores con un porcentaje de masa muscular (MM) mayor, el peso de carga capaz de manipular es significativamente mayor. El peso máximo aceptable para realizar las tareas de levantamiento, utilizando metodología Snook y Ciriello, fue de 18,33 kg, reduciéndose ésta a 15,58 kg cuando la carga no cuenta con asas. Respecto a las dolencias musculoesqueléticas, se observó una alta frecuencia de malestares a nivel lumbar y dorsal, como consecuencia de posturas de trabajo inadecuadas al hacer levantamiento manual de cargas durante toda la jornada de trabajo. 


\section{REFERENCES}

1. Luttmann A, Jager M, Griefahn B, Caffier G, Liebers F, Steinberg U. Preventing Musculoskeletal Disorders in the Workplace [on line]. Geneva: WHO; 2003 [cited 2014 Nov]. 32 p. (Protecting Workers' Health Series; 5). Available from: http://www.who.int/occupational_health/publications/en/ oehmsd3.pdf

2. Instituto Nacional para la Seguridad y Salud Ocupacional-NIOSH. Cómo prevenir los trastornos musculoesqueléticos [en línea]. Atlanta: CDC; 2012 [citado nov 2014]. (Datos breves de NIOSH; 2012-120). Disponible en: http://www.cdc.gov/spanish/niosh/docs/2012-120_sp/

3. Punnett L, Pruss-Ustun A, Nelson D, Fingerhut M, Leigh J, Tak S, et al. Estimating the global burden of low back pain attributable to combined occupational exposures. Am J Ind Med. 2005; 48(6):459-469.

4. Asociación Chilena de Seguridad-ACHS. Enfermedades del Sistema Musculoesquelético en la Industria de la Construcción [en línea]. Santiago de Chile: ACHS; 2007 [citado 8 Nov 2014]. Disponible en: https://edoc.site/tmeconstruccion-achs-web-pdf-free.html

5. Chile. Ministerio del Trabajo y Previsión Social. Guia Técnica para la evaluación y control de los riesgos asociados al manejo o manipulación manual de carga. Resolución Exenta No. 22 [en línea]. Santiago: Ministerio del Trabajo y Previsión Social; 2018 [citado feb 2018]. Disponible en: https://www. previsionsocial.gob.cl/sps/guia-tecnica-la-evaluacion-control-riesgosasociados-al-manejo-manipulacion-manual-carga/

6. Chile. Ministerio del Trabajo y Previsión Social. Modifica el código del trabajo para reducir el peso de las cargas de manipulación manual. Ley 20.494. Santiago: Ministerio del Trabajo y Previsión Social; 2016 [citado 25 jul 2017]. Disponible en: https://www.leychile.cl/Navegar?idNorma=109489 9\&idParte $=$ \&idVersion $=2016-09-17$

7. Waddell G, Burton A. Occupational health guidelines for the management of low back pain at work: evidence review. Occup Med (Lond). 2001; 51(2):124-135.

8. Cerda C. Estudio comparativo de la composición corporal basado en métodos de antropometría y bioimpedanciometría [tesis mag]. Temuco: Universidad de La Frontera; 2010. p. 10-11.

9. Becker JP. Las Normas ISO 11228 en el Manejo Manual de Cargas. XV Congreso Internacional de Ergonomía SEMAC [en línea]. México: SEMAC; 2009 [citado 7 ene 2015]. Disponible en: http://www.semac.org.mx/ archivos/congreso11/Pres09.pdf

10. Córdova V, Troncoso R, Pinto R. Percepción del Peso de una Carga en Población Laboral Masculina Chilena: Modelamiento Basado en la Teoría de Conjuntos Difusos. Cienc Trab. 2010; 12(37):370-375.

11. Córdova V, Pinto R, Llambias J, Chávez B. Capacidad de Manejo Manual de Carga de Trabajadores Chilenos: Pesos Máximos Aceptables para Tareas de Levantamiento. Cienc Trab. 2009; 11(34):204-210.

12. Córdova V, Troncoso R, Pinto R. Comparación de la Percepción del Peso de una Carga en Población Laboral Femenina: Sector Industrial Versus Sector Salud. Cienc Trab. 2011; 13(39):17-23.

13. Ruiz L. Manipulación Manual de Cargas. Tablas de Snook y Ciriello. Norma ISO 11228. Madrid: Instituto Nacional de Seguridad e Higiene en el Trabajo; [fecha desconoc] [citado 19 feb 2015]. Disponible en: http://www.google.cl/ url?sa $=$ t\&rct $=j \& q=\&$ esrc $=s \&$ source $=$ web $\& c d=1 \& v e d=0 C B w 0 F j A A \& u r l=$ http $\% 3 \mathrm{~A} \% 2 \mathrm{~F} \% 2 \mathrm{Fwww.insht.es} \% 2 \mathrm{FMusculoEsqueleticos} \% 2 \mathrm{FContenidos} \% 2$ FFormacion\%2520divulgacion\%2Fmaterial\%2520didactico\%2FSyC_ ISO\%252011228.pdf\&ei=DBOfVdWjKcPesAWE2YGYBw\&usg=AFOjCNGgwL trifPMzNUURttDgaDjifwoTw\&bvm=bv.89947451,d.eXY\&cad=rjt

14. Ministerio del Trabajo y Previsión Social. Guía Técnica para la Evaluación del Trabajo Pesado [en línea]. Santiago: Gobierno de Chile-Superintendencia de Pensiones; 2010 [citado 12 nov 2014]. Disponible en: https://www.hysla. com/guia-tecnica-para-la-evaluacion-del-trabajo-pesado/

15. Marugán $P$, Precioso J, Zorrilla G, Ferrete $M$, Val Cacho M. Prevención de riesgos laborales en los trabajadores del sector del transporte; Parte I. Valencia: Servicio de Prevención de la Empresa Municipal de Transportes de Valencia; [fecha desconoc] .

16. Lohman T, Roche A, Martorell R. Anthropometric standardization reference manual. Champaign: Human Kinetics Books; 1988. 199 p.

17. Ruiz L. Manipulación manual de cargas. Guía técnica del INSHT [en línea]. Madrid: Instituto Nacional de Seguridad e Higiene en el Trabajo; 2011 [citado 25 jun 2015]. Disponible en: http://www.insht.es/MusculoEsqueleticos/Contenidos/Formacion\%20divulgacion/material\%20didactico/ GuiatecnicaMMC.pdf

18. Snook S, Ciriello V. The design of manual handling task: Revised tables of maximum acceptable weights and forces. Ergonomic. 1991; 34:1197-1213.

19. Ayoub $M$, Dempsey P. The psychophysical approach to manual materials handling task design. Ergonomics. 1999; 42(1):17-31.

20. Vergara M. Evaluación ergonómica de sillas. Criterios de evaluación basados en el análisis de la postura [tesis]. Castellón de la Plana: Universitat Jaume I; 1988. p.11.

21. Burkhalter N. Evaluación de la escala de Borg de esfuerzo percibido aplicada a la rehabilitación cardiaca. Rev Latino-Am Enfermagem. 1996 [citado15 mar 2015]; 4(3):65-73. Disponible en: http://www.scielo.br/scielo.php?pid= S0104-11691996000300006\&script=sci_arttext

22. Corlett E, Bishop R. A technique for assessing postural discomfort. Ergonomics. 1976; 19(2):175-182.

23. Rueda M, Zambrano M. Manipulación manual de cargas. En: Rueda $M$, Zambrano M. Manual de Ergonomía y seguridad. México, D.F.: Alfoaomega; 2013. p. $14-35$.

24. España. Instituto Nacional de Seguridad e Higiene en el Trabajo. Guía técnica para la evaluación y prevención de los riesgos relativos a la manipulación manual de cargas, Real Decreto 487/1997, 14 abr B.0.E. n 97, 23 abr [en línea]. Madrid: INSHT; 2003 [citado 25 jul 2017]. Disponible en: http:// www.insht.es/InshtWeb/Contenidos/Normativa/GuiasTecnicas/Ficheros/ cargas.pdf.

25. Chile. Ministerio de Salud. Norma Técnica de identificación y evaluación de factores de riesgo asociados a trastornos musculoesqueléticos relacionados al trabajo (TMERT) de extremidades superiores. Santiago: MINSAL; 2012 [citado 12 nov 2014]. Disponible en: http://web.minsal.cl/portal/url/item/ cbb583883dbc1e79e040010165014f3c.pdf 